\title{
THE RELATIONSHIP OF VISUAL EVOKED POTENTIALS TO CORTICAL PHYSIOLOGY
}

\author{
Ken Nakayama \\ Smith-Kettlewell Institute \\ of Visual Sciences \\ San Francisco, California 94115
}

The last 15 years have seen a rapid expansion in studies on human visual evoked potentials (VEP), both at the fundamental and at the clinical level. A wide variety of stimulus conditions have been explored, ${ }^{1}$ new types of mathematical tools have been introduced, ${ }^{2,3}$ and there has been an increasing appreciation of possible VEP correlates with underlying physiological mechanisms. ${ }^{4}$ Variability of the folding and size of the human striate cortex ${ }^{5}$ as well as the remote location of human scalp electrodes, however, limits the accurate localization of human VEP components to particular cortical structures. In this regard it would seem desirable to explore the VEP of a laboratory animal closely related to the human.

In this paper I discuss two approaches that may prove both useful and complementary in deciphering visual evoked potentials in monkey, and by extrapolation, in man. The first approach involves the use of fine stimulus control, noting the degree to which the VEP amplitude varies with small changes in stimulus characteristics. The second approach, still in the preliminary stages, involves the use of current source density analysis, a method to localize the generating processes of the VEP to specific cortical laminae and sublaminae.

\section{Stimulus Control of Spatial and Temporal Frequency}

The ability of the human observer to see variations in brightness, either in time or in space, has been measured psychophysically by obtaining the temporal or the spatial modulation sensitivity functions, respectively. In time, this involves the plotting of the reciprocal of threshold modulation (the sensitivity) required to see flicker at various temporal frequencies. ${ }^{6}$ In space, it involves the measurement of sensitivity for different spatial frequencies of sine wave gratings. The curve relating contrast sensitivity to spatial frequency is a broad unimodal one (the dashed line in FIGURE 1), peaking around 5 cycles/deg. ${ }^{7}$ Rather than being determined by a single mechanism, there is good evidence to suggest that this contrast sensitivity function reflects the upper envelope of sensitivities of many different subsystems, each tuned to a much more narrow range of spatial frequencies.

Psychophysical evidence indicates that one can adapt a spatial frequency "channel," having a tuning function approximating a bandwidth of about 1 octave, much more narrow than the overall contrast sensitivity function. ${ }^{8}$ Electrophysiological evidence provides a biological substrate for such channels. Cortical cells have a narrow range of preferred spatial frequencies, much narrower than that reflected by the psychophysical contrast sensitivity function. The center frequency of each cell can

*This work was supported in part by Grants SROI EY-01582, 5RO1 EY-02124, SP30 EY-01186, and 2SO RR 05566 from the National Institutes of Health and by the SmithKettlewell Eye Research Foundation. 
vary over quite a range. FIGURE 1 shows the relationship between the tuning function of individual cortical cells as obtained by DeValois, et al. ${ }^{9}$ in relation to the psychophysical curve. The range of spatial frequency tunings of individual cortical cells is consistent with the overall sensitivity curve obtained psychophysically.

What should be expected if we were to record from the surface of the skull of the human or monkey, plotting VEP amplitude as a function of spatial frequency? If there were many narrowly tuned mechanisms, each tuned to a slightly different spatial frequency and if each mechanism or cell type could be recorded with approximately equal weighting on the surface of the scalp, then one might expect that the contrast evoked potential results would mirror those obtained by psychophysics.

In fact, the first systematic investigation relating VEP to spatial frequency showed such a relation..$^{10}$ By presenting counterphase-modulated sinusoidal gratings to human observers (essentially equivalent to exchanging the bright and dark bars of the grating) at a reversal rate of $16 \mathrm{~Hz}$, Campbell and Maffei noted a linear relationship between VEP amplitude and log contrast of the grating. Extrapolating this linear function to zero $\mu \mathrm{V}$, they obtained a VEP estimate of the contrast threshold, one that appeared rather close to that obtained by psychophysics. These results suggested that the VEP indeed refiects the psychophysics and that by making VEP measurements one could hope to tap the envelope of activities of highly representative sets of visual cortical neurons. As such, the VEP could be considered as an alternative technique to obtain results that could be obtained psychophysically.

Upon closer examination, using much more resolution and over a wider range of conditions, however, we have shown that the human steady-state VEP has perhaps the opposite characteristic." Rather than being broadly tuned to spatial frequency, it was generally very sharply tuned (FIGURE 2) and with great reproducibility. Furthermore, these spatial frequency tuning functions were highly dependent on temporal frequen-

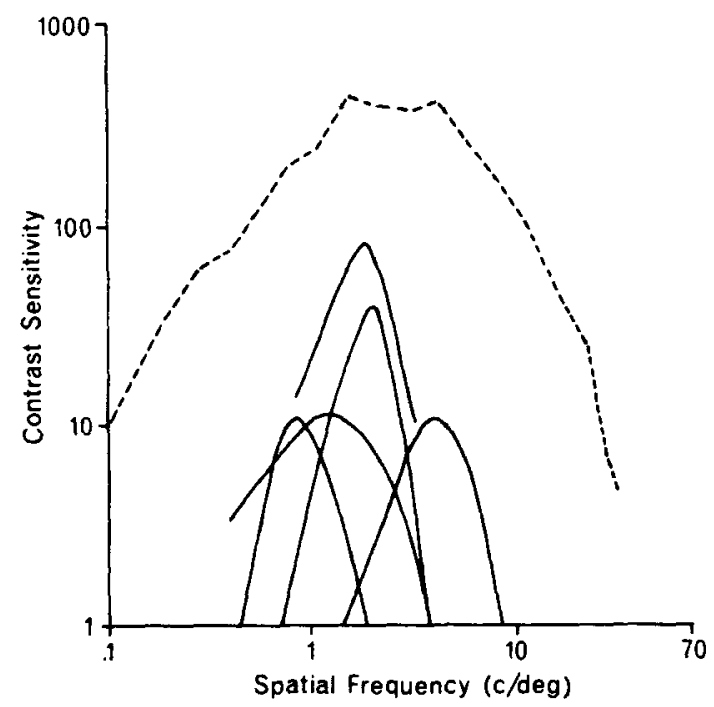

FigurE l. Upper dashed curve is the human psychophysical contrast sensitivity function. Sensitivity (defined as the reciprocal of theshold) is plotted as a function of spatial frequency for vertical sine wave gratings. (After Campbell and Robson.') Lower solid curves represent contrast sensitivity functions obtained from visual cortex single units. (After DeValois et al. ${ }^{9}$ ) 


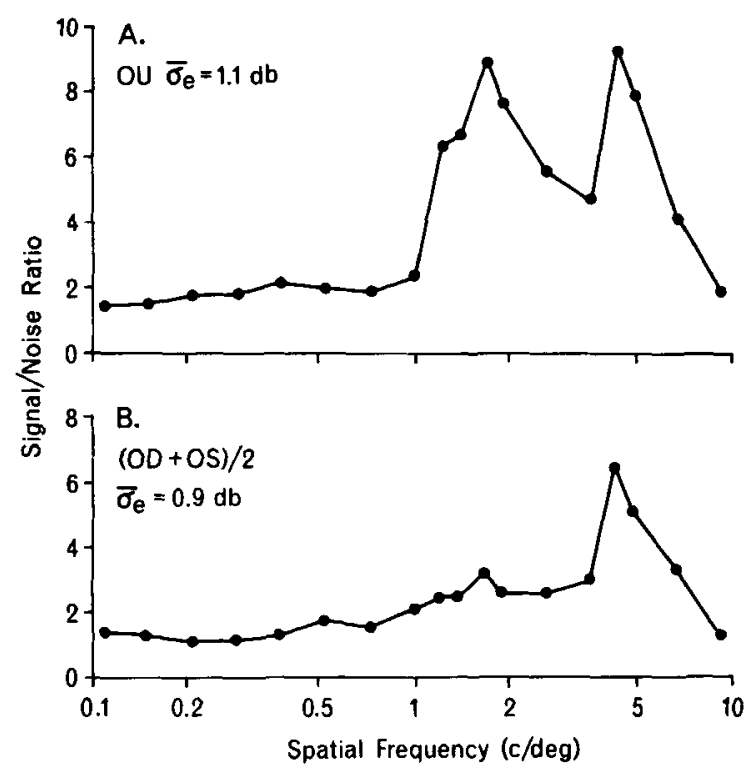

FIGURE 2. (A) Human VEP amplitude versus spatial frequency for binocularly presented gratings. Counterphase reversal rate and recording frequency was $30 \mathrm{~Hz}$. Contrast was $68 \%$. (B) Same as above except for monocular viewing. (After Apkarian, Nakayama \& Tyler. $^{29}$ )

cy. Thus, rather than speaking of a simple spatial or temporal frequency function, it is more accurate to speak of a multiple peaked spatial/temporal frequency surface (FIGURE 3).

Steady-state surface VEPs recorded in alert cats and alert monkeys revealed a qualitatively similar picture. ${ }^{12,13}$ For example, in the alert monkey, trained to fixate a spot to receive a water reward, the spatial and temporal frequency tuning was as sharp or sharper than that seen for humans (FIgURE 4). Note that in this figure, the amplitude versus spatial frequency tuning function is a very sharply peaked one, centered on 10 cycles $/ \mathrm{deg}$, and that, for at least 4 electrode placements, there is essentially no response below about 3 cycles/deg. Thus, for surface recording from this monkey, the spatial frequency tuning function is as narrow as that seen for individual cortical neurons in the same species. They compare well with the results of DeValois et al., ${ }^{9}$ as seen in FIGURE 1 and more directly with the results of Schiller et al., ${ }^{14}$ who plotted spike counts as a function of spatial frequency. Tuning in the temporal frequency domain was also very sharp for the monkey VEP. FIGURE 5 shows the relation between temporal frequency and the VEP amplitude for 4 spatial frequencies, also showing the progressive variation of phase lag with increasing temporal frequency.

The existence of very narrow spatial frequency tuning in the VEP raises a wide range of questions. Why was it not seen by Campbell and Maffei ${ }^{10}$ ? Is it the only response that can be obtained? What does it mean in terms of the genesis of these steady-state visually evoked potentials?

Campbell and Maffei's resuits were restricted to very few electrode placements and the results of only one temporal frequency were reported. Our own results indicate that results like that found by Campbell and Maffei can be obtained both in the 
A.

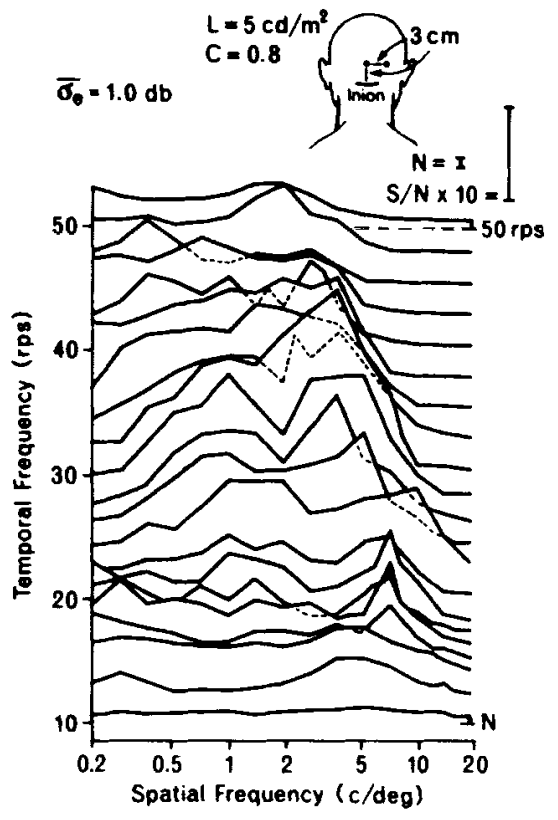

S.A.

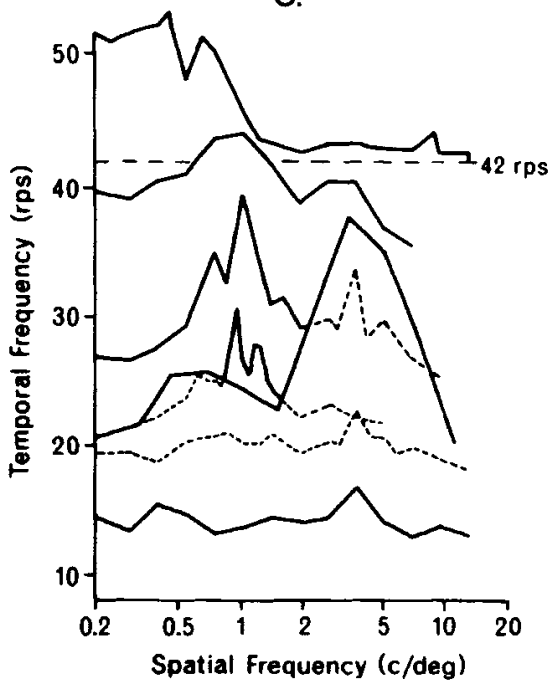

B.

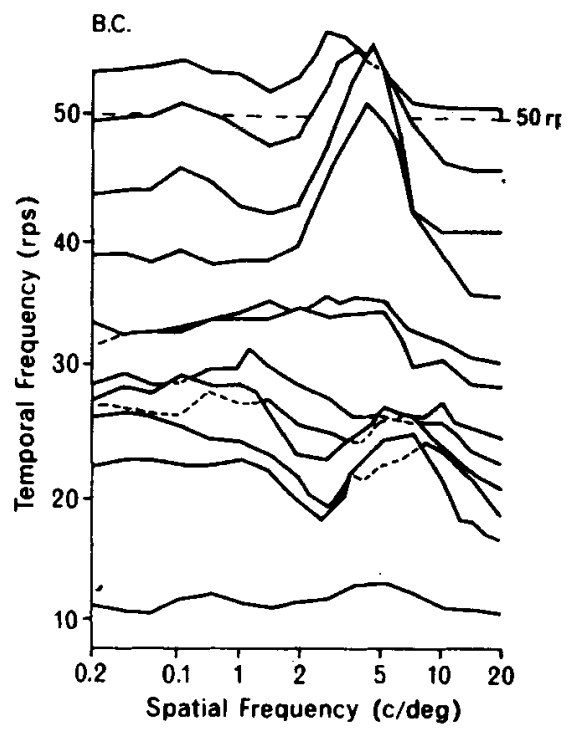

D.

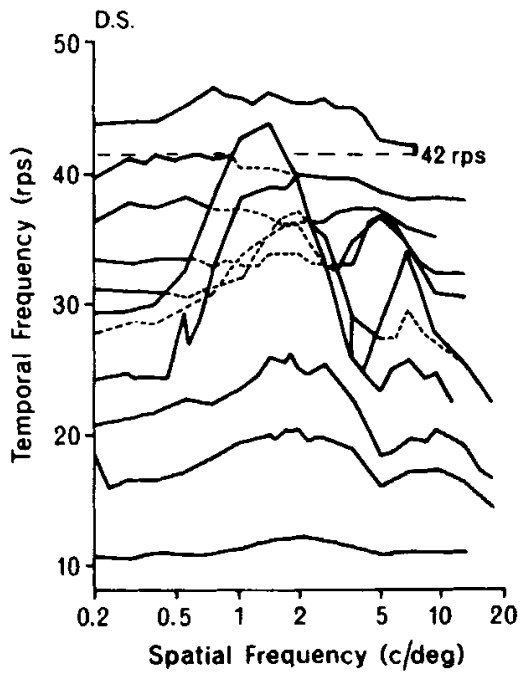

Figure 3. Spatial frequency tuning as a function of temporal frequency (rps) for four different human observers (A-D). Each curve is plotted from a baseline at its frequency specified on the ordinate with VEP amplitude plotted as an EEG signal/noise ratio at the recording frequency. Note the high degree of stimulus specificity in both the temporal and the spatial frequency domains. Although the details differ for each observer at the same electrode position, multiple narrow spatial frequency tuning is prevalent. (After Tyler, Apkarian \& Nakayama. ${ }^{11}$ ) 
human and in the monkey, but they are far from the rule. For example, FIGURE 6A shows the contrast responses of a single electrode (electrode 2 seen in Figure 4), associated with an extrapolated VEP sensitivity curve for the monkey. First it should be noted that although the contrast functions are linear over quite a range, they often have two limbs, a relatively shallow section at low contrast and a steeper sloped section at higher contrast. Either function can occur in combination or alone (see below). Although the steep high contrast function has been reported previously, ${ }^{10}$ it has

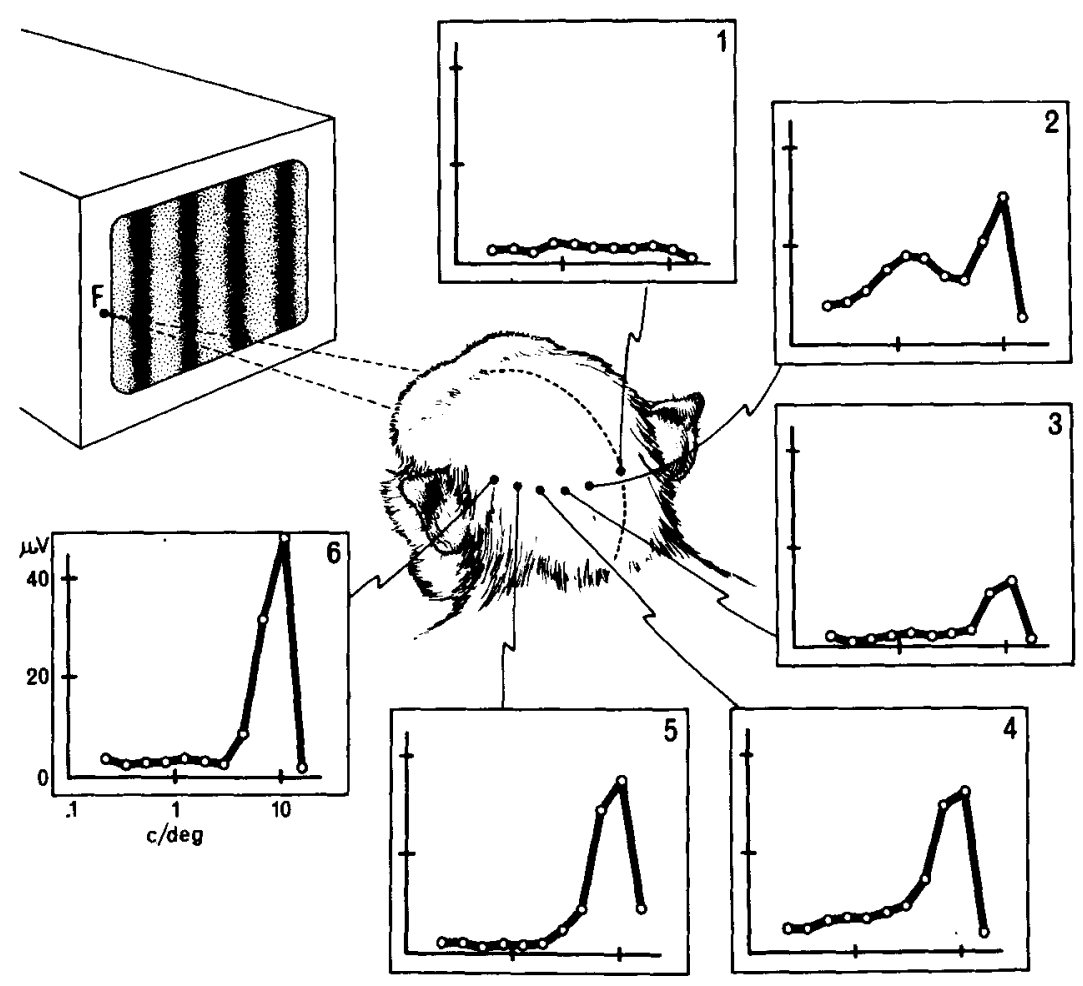

FIGURE 4. Spatial frequency tuning of the steady-state VEP in the alert primate, trained to fixate on a spot (point F). Responses are obtained from 6 epidural electrode placements, localized along the occipital region of monkey cortex. Foveal representation of the striate cortex is represented in the more anterior placements. Note the high degree of spatial frequency tuning for nearly all of the electrode channels. Contrast is $33 \%$. Counterphase reversal rate and recording frequency are maintained at $16 \mathrm{~Hz}$. (From Nakayama, Mackeben \& Sutter. ${ }^{13}$ )

elicited little interest. Its existence, however, indicates a separate pattern sensitive mechanism as yet to be revealed by single unit or psychophysical techniques.

Extrapolating the lower contrast threshold curve to zero $\mu \mathrm{V}$ (arrows in Figure 6A) yields a set of extrapolated thresholds (dots) which can be compared with the monkey psychophysical data. This can be seen in FIGURE 6B. The fit of the extrapolated monkey VEP to monkey psychophysics is rather good, both in the shape and the absolute magnitude of the sensitivity curves. 

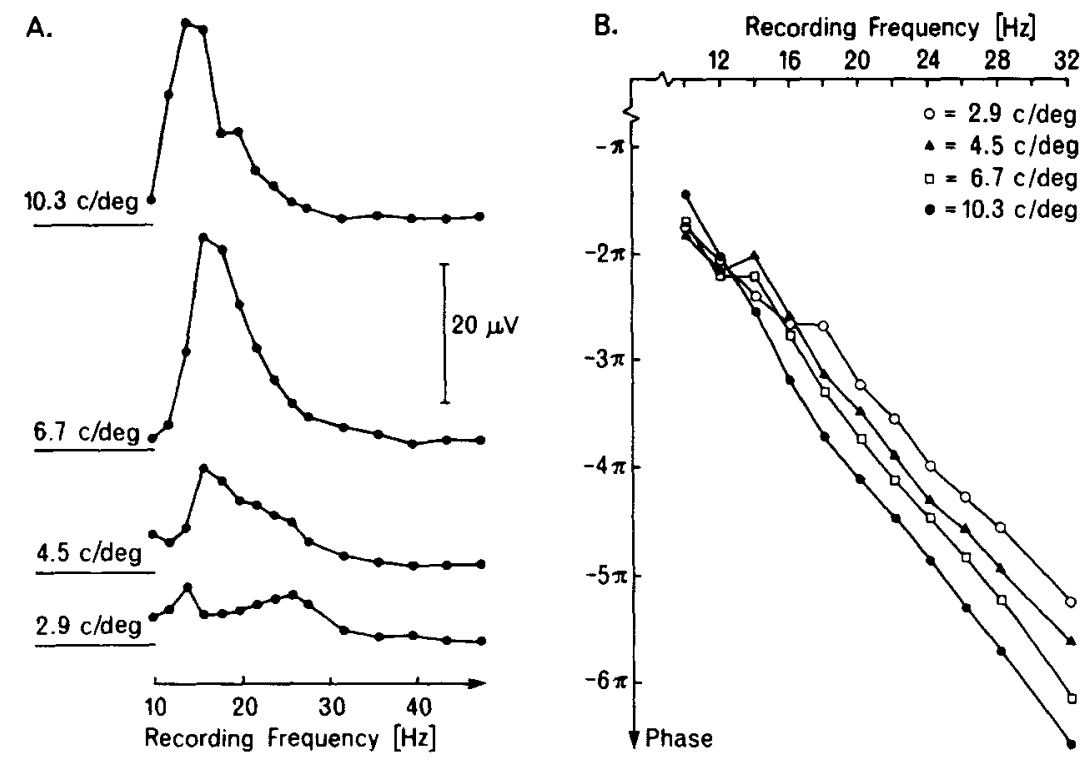

Figure 5. (A) Temporal frequency tuning for 4 spatial frequencies in an alert monkey obtained from electrode 6 (as shown in Figure 4). (B) Corresponding phase lag at different temporal frequencies. (From Nakayama, Mackeben \& Sutter. ${ }^{13}$ )

This is a rare example, however, of such a close match. Under other circumstances the agreement is much worse. Note that in FIGURE 7, the extrapolated VEP curve bears no similarity to the psychophysics. The reason for this poor fit is important to note. It does not fit because at electrode 3, 4, 5, and 6, (as depicted in FIGURE 4) there is no low-frequency contrast portion of the VEP versus log contrast function, leaving only the steep higher contrast curve. Extrapolating this remaining steep higher contrast to zero $\mu \mathrm{V}$ leads to a high VEP threshold which does not fit the psychophysics. Thus, it should be of no surprise that the VEP versus spatial frequency curve will show some large departures from the psychophysics. The fit will depend heavily on which contrast function is present and obviously on the contrast that is used to obtain the spatial frequency function. FIGURE 6C (bottom right panel), for example, shows the change in spatial frequency tuning obtained from a single electrode placement for 3 different contrast levels. As contrast is increased, one reaches the steeper higher contrast portion of just some of the functions shown on the left of FIGURE 6, and, as a consequence, the spatial frequency tuning is much more sharply tuned.

Before suggesting a neurophysiological framework to interpret the above results, one needs to establish that such a framework is indeed necessary; that the responses, for example, are not due to an electrical artifact, namely, the cancellation of opposing polarity signals from more broadly tuned mechanisms. This purely electrical interpretation seems unlikely for several reasons. First, the temporal phases of the two peaks in the human spatial frequency response were often very close and there was no indication that many of the multiple peaks could be explained by opposite polarity responses canceling. "Second, under some conditions individual peaks could disappear almost completely without affecting other peaks. This was especially prominent for some cases of monocular versus binocular stimulation as shown in FIGURE 2. Finally, 
the monkey results often showed only one very sharp spatial frequency peak (as seen in FIGURE 4) at relatively high spatial frequencies. Thus, there was no second mechanism that could electrically cancel to result in the narrowly tuned peak.

The similarity between the steady-state pattern VEP seen in the human and that in the monkey is noteworthy, especially in light of the very large differences in the gross layout of the striate and parastriate cortex in each species. In humans the

A.
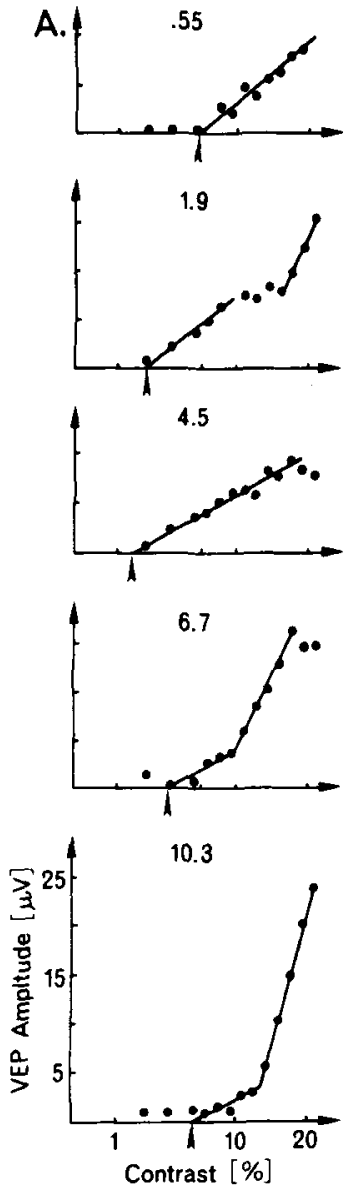

B.
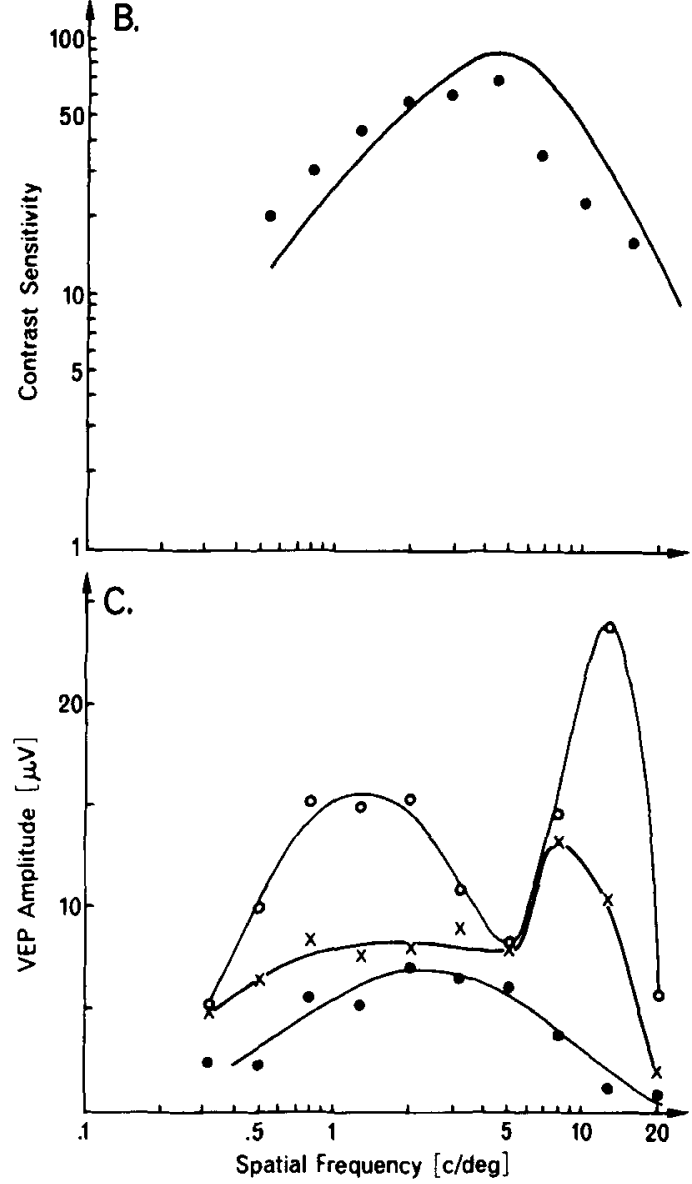

FIGURE 6. (A) Monkey VEP amplitude (plotted on a linear scale) vs grating contrast (plotted on a logarithmic scale) for 5 different spatial frequencies as noted by numerals above each function. Arrows denote the position of the extrapolated VEP threshold. (B) Contrast sensitivity of the VEP as derived from VEP thresholds (solid dots). VEP recordings were obtained from electrode 2 as shown in FIGURE 4 (from Nakayama et al. ${ }^{31}$ ). As a comparison, corresponding monkey psychophysical data is presented as the smoothed solid curve (from DeValois et al. ${ }^{32}$ ). (C) VEP amplitude vs spatial frequency function for 3 different contrasts (dots $=9 \%$; crosses $=$ $18 \%$; open circles $=33 \%$ ). (From Nakayama \& Mackeben. ${ }^{33}$ ) 


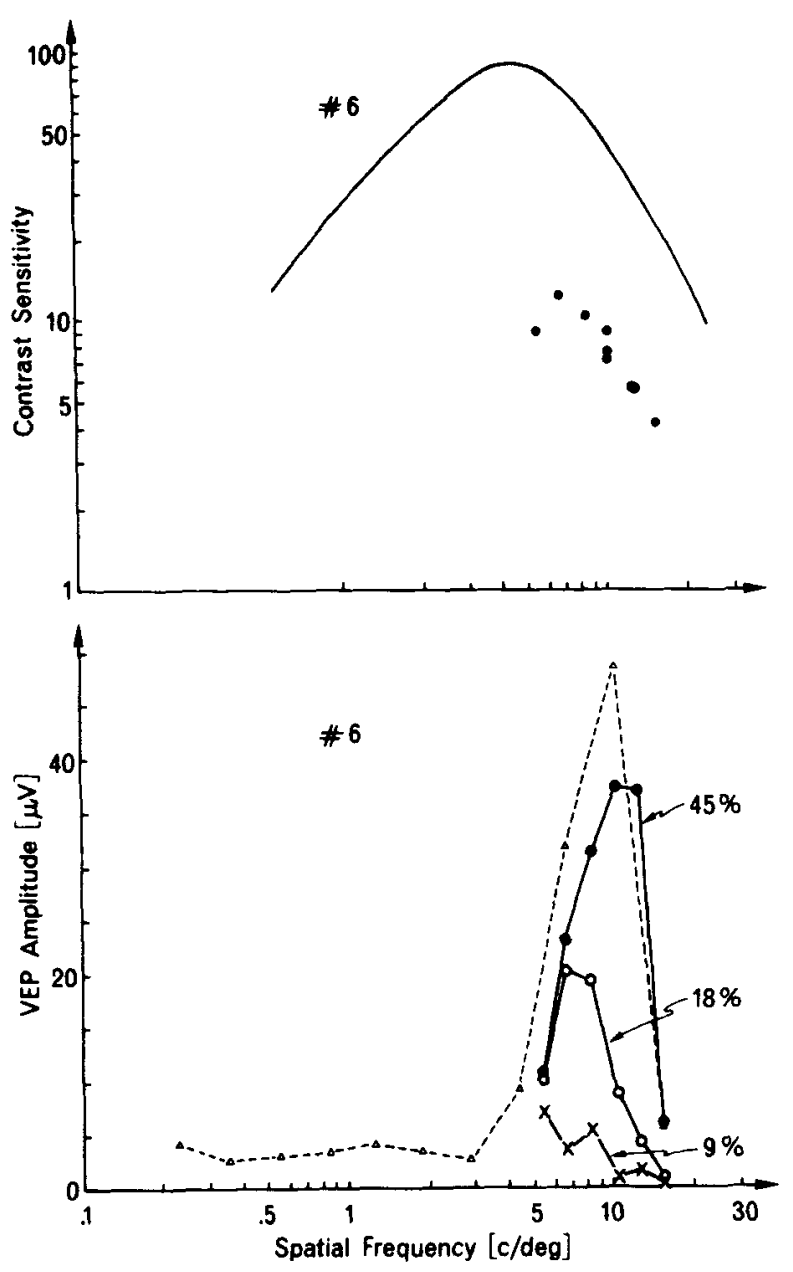

Figure 7. Same conditions as shown in Figure 6 except for a different electrode in the same monkey. Upper graph: VEP contrast sensitivity (dots) as compared with monkey psychophysical results (solid line). Note the very poor match of VEP and psychophysics. Lower graphs show the VEP amplitude vs spatial frequency for 3 levels of contrast (From Nakayama \& Mackeben. ${ }^{33}$ )

primary cortex is mostly buried within the mesial walls of the posterior lobe and within the calcarine fissure. In contrast, the secondary cortex is exposed. In the macaque, it is almost the reverse, with much of the striate cortex exposed over the posterior cortical region and the parastriate fields buried. ${ }^{15}$ The similarities of the steady-state pattern VEP in both man and monkey, especially in light of these gross anatomical differences, indicates that it must be the microanatomical or microphysiological aspects of the cortex that lead to this degree of VEP specificity.

Although many interpretations are possible at this microanatomical level, one broad conclusion seems indicated, namely, that the surface VEP records only a fraction of the cortical neurons which might be thought to respond to the stimulus and 
which could be expected to be involved in perception. For example, consider the human curves seen in Figures 2 and 3 and the monkey data seen in Figure 4. For these electrode placements, no VEP is evident over at least some range of spatial/ temporal frequencies that are visible to the person or the animal. One hypothesis to explain such a result is that of neural "resonance." This hypothesis suggests that the repetitive nature of the stimulus required to obtain a steady-state VEP brings out narrow tuning characteristics in certain sets of cortical neurons. For example, neurons having certain spatial characteristics (namely, a given receptive field size) might also share similar temporal characteristics, similar time constants in feedback loops, cable constants, and so on. As such, they may be extremely selective to the temporal frequency of the stimulus. By using the term "resonance" rather loosely, it is not assumed that it is identical to the concept of resonance used in linear theory. In fact, many of the narrow temporal peaks are likely to be due to nonlinear processes. Before considering this hypothesis in further detail, however, it would be best to consider the results of another relevant neurophysiological technique, that provided by the measurement of the VEP at different cortical depths.

\section{Current Source Density Analysis}

A second possible approach in the search for a physiological interpretation of evoked potentials is that of current source density analysis, a technique which has had a long history, ${ }^{16}$ but which has only recently become applicable to the VEP. This endeavor has been furthered most recently by a pair of tutorial papers ${ }^{17,18}$ followed by an important set of papers elucidating the electrically elicited field potentials obtained in various visual structures by Mitzdorf and Singer. ${ }^{19-21}$

Because the mathematical reasoning, the assumptions, and the description of the CSD technique are well described in the above papers, the reader is urged to consult them for details. In essence, the approach can be seen as having several aspects. First is the application of electromagnetic theory to the field potential distribution in neural tissue. If one assumes homogeneity and horizontal translation symmetry in two dimensions, a one-dimensional current source/sink distribution can be obtained by estimating the spatial second derivative of potential with respect to cortical depth. As such, this first part of the endeavor is an exercise in applied physics and is relatively straightforward. It estimates the net sink or source contribution for particular cortical depths. A more difficult problem is to provide an interpretation of the sink/source distributions in terms of neuronal activity. This latter task is not straightforward, and, as has been pointed out by others, ${ }^{22}$ it requires a wealth of additional knowledge, including the detailed anatomy and physiology of cortical tissue.

The measurement of field potentials at different depths of cortex can be obtained in several ways. Early experiments advanced the electrode in small increments to the cortex, measuring the field potentials at successive depths. More recently, many laboratories are beginning to use multiple electrode arrays, either using metal electrodes or multiple pipettes, as reported by Vaughan in this volume. ${ }^{23}$ The primary work in visual structures was collected by using electrical stimulation of the afferent tracks. ${ }^{19-21}$ Of significance is the fact that an $X, Y$ system as inferred from conduction velocities could be segregated at the level of cortex in cat and monkey. Furthermore, new X, Y subdivisions, not previously described, were noted in the LGN of cat ${ }^{19}$ and now confirmed by intracellular HRP injection techniques.$^{24}$ Of particular importance to those interested in the interpretation of the surface VEP is the relevance of the "open" versus "closed" field current generators (as originally described by Lorente de No. ${ }^{25}$ ) It determines whether a given set of synaptic currents in the depths will be 
visible as a surface VEP. For example, electrical stimulation of area-18 afferents in the cat leads to a prominent surface positivity which is the result of sinks in layer IV and adjacent sources above. ${ }^{20}$ This dipolar sheet configuration generates large potentials even as far away as the cortical surface. With the electrically evoked activity in the monkey striate cortex, however, it appears that the potential deflections seen at the cortical surface is but a pale reflection of the voltages seen in the depths. ${ }^{21}$ Current source density analysis of these monkey field potentials suggests a reason for this difference, showing the predominance of "closed field" sink/source distributions in the striate cortex of the monkey, at least for electrical stimulation. Closed field source/sink distributions are not dipolar, but are "sandwich" like, showing a sink bounded by sources above and below. As a result, these currents do not generate appreciable potential deflections at distant sites.

Our preliminary studies of the monkey striate cortex in response to visual rather than electrical stimulation ${ }^{26}$ reveal additional features. In brief, the recording electrode consisted of a set of 16 insulated stainless steel wires ( $50 \mu \mathrm{m}$ in diameter) arranged in a parallel array. Ends are cut so as to provide a vertical contact separation of $150 \mu \mathrm{m}$ between adjacent recording poles. With our electronically matched 8-channel amplifier system, every other contact could be recorded simultaneously and the stimulus was then repeated for the recording from the 8 other channels. Because the differentiation grid used for the current source density calculations was $300 \mu \mathrm{m}$, all CSDs were calculated from field potentials obtained simultaneously, removing one of the most important sources of extraneous variability.

In FIGURE 8 we see the field potentials obtained for two intensities of stroboscopic flash for different cortical depths. FIGURE 9 shows the corresponding CSD profiles. Several aspects should be noted. First is the fact that the predominantly positive surface potential is very small compared to the large negative potentials seen in the depths. Note the degree to which the sinks and sources are spatially localized by the CSD analysis and the fact that there is great consistency of this sink source distribution when comparing high versus low intensity flash (compare the right versus left column in Figure 9). In both cases, the earliest response is a set of two prominent sinks, the lowest most probably in layer IV (see later), each bordered by a prominent source above. This is then followed by a sequence of current source densities of opposite polarity at the same depth. Small though consistent indications of an early and later sink can also be seen in the bottom CSD tracings, possibly reflecting excitatory activity of layer VI afferents. The main difference between the two intensities of flash is the duration and latency of the deflections. Although the response of the dimmer flash is smaller and slower, it has the same spatial structure. Thus the neuronal elements activated by dim flash appear as nearly identical to those activated by the higher intensity flash.

Two general features of the data deserve further emphasis. First is the fact that in comparison to the depths, the potentials seen at the surface are very small. Second is the rather complex, and yet reliable, pattern of sources and sinks that can be derived from these potentials. This indicates the existence of a "closed field" situation mentioned earlier although it does not prove that the individual neurons themselves have closed field characteristics. In particular, the synaptic currents of several neuronal classes might summate to generate a net cancellation of current when seen from the surface. These results indicate that at least for strobe flash in monkey striate cortex, the surface evoked potential is a poor indicator of cortical activity immediately below the electrode.

So far we have confined ourselves to the localization of net transmembrane current sources and sinks from a CSD analysis of cortical field potentials. This leaves unanswered the question as to the physiological interpretation of such CSD profiles. To start, Mitzdorf and Singer have made a bold hypothesis. ${ }^{19}$ They argue that 

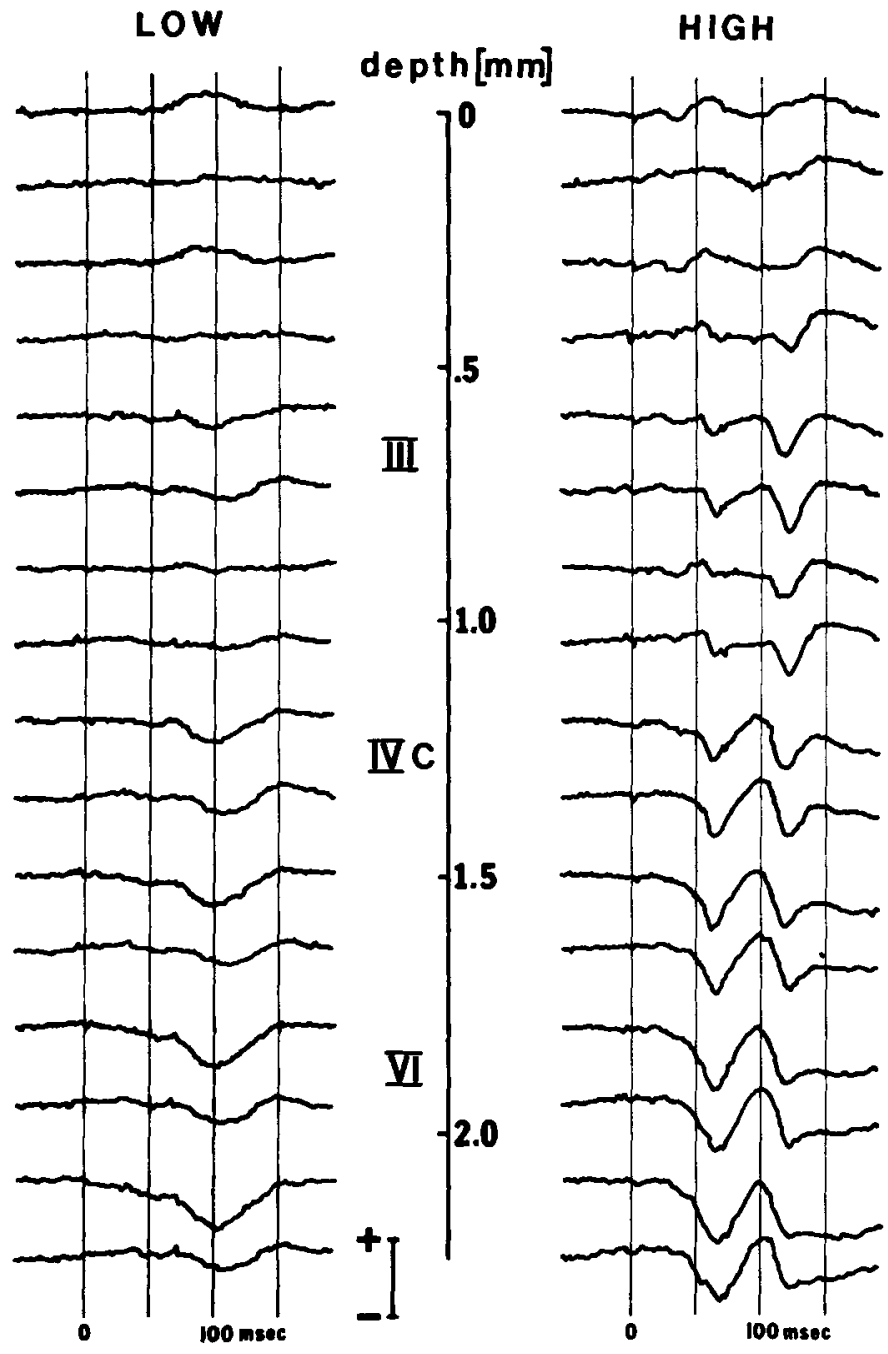

FIGURE 8. Transient VEP profiles in monkey striate cortex for 2 intensities of strobe flash. Potential functions were obtained for 16 depths in cortex spaced at $150 \mu \mathrm{m}$. Each time-averaged response is the mean of 64 sweeps. Roman numerals represent the estimated location of key cortical laminae. Voltage calibration is $100 \mu \mathrm{V}$. Flash begins at vertical line marked by zero. Distance between time markers is $50 \mathrm{msec}$. Animal is sedated with light Nembutal anesthesia. (From Mackeben, Mitzdorf \& Nakayama. ${ }^{26}$ )

essentially all electrically elicited field potentials in the visual system, including surface potentials, are reflections of excitatory synaptic currents. Thus the distribution of sinks indicates the relative strength of excitatory synapses whereas the distribution of sources primarily reflects the associated outward loop closing currents adjacent to these excitatory synapses. As such, inhibition in the form of IPSPs makes a negligible contribution of field potentials and associated CSDs in the visual cortex.

In brief, Mitzdorf and Singer's argument runs as follows: ${ }^{19}$ Extracellular voltage 
differences are due to extracellular current flow, which in turn originates from transmembrane current flow. Most current fow in neural tissue is capacitative. Thus, transmembrane current is roughly proportional to the first time derivative of membrane potential. Because the slope of the rising phase of EPSPs of visual neurons is about ten times greater than for IPSPs, EPSPs contribute much more to the extracellular current flow. This difference is further exaggerated by the fact that the
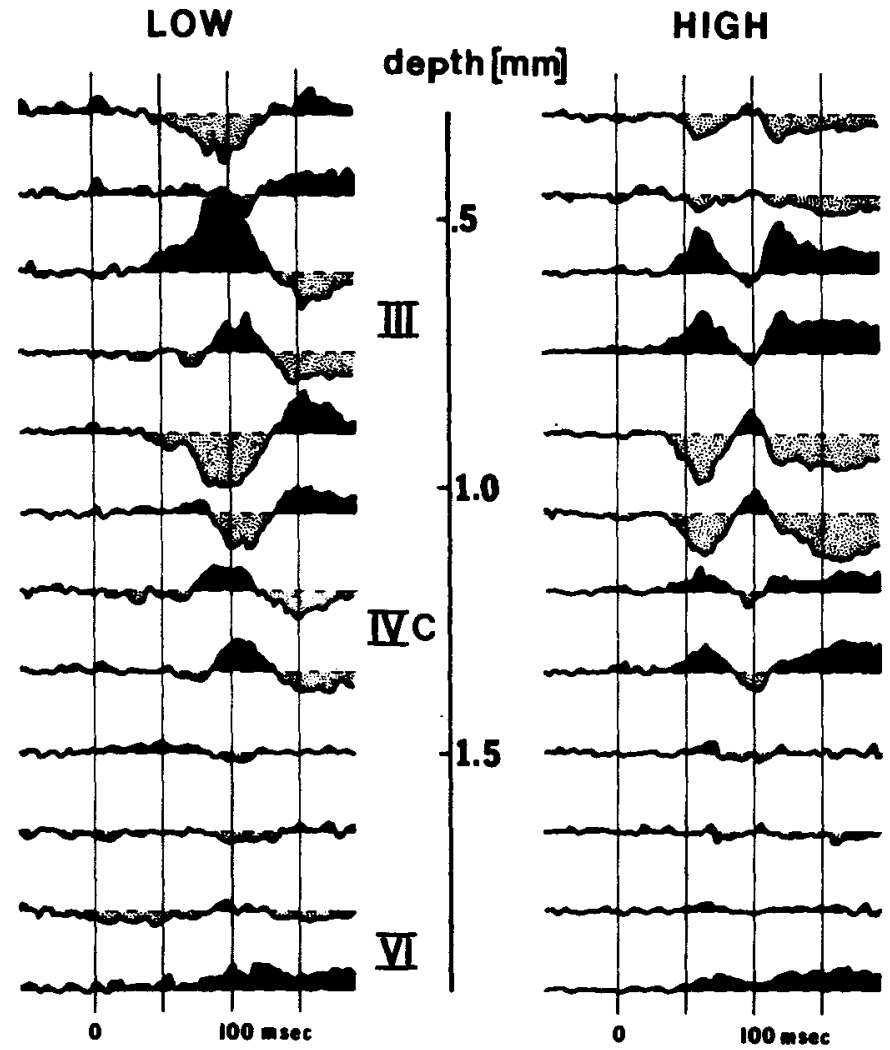

Figure 9. Corresponding current source density profiles obtained from the monkey field potentials presented in FIGURE 8. Current source density is plotted as a function of time for 14 depths in cortex. Sinks (net inward transmembrane current density) are depicted by upward deflections and are identified as solid areas. Sources (net outward transmembrane current density) are represented by downward deflections and are filled in as dotted areas. The magnification of the low-intensity flash CSD profiles is twice that as pictured for the higherintensity flash. (From Mackeben, Mitzdorf \& Nakayama. ${ }^{26}$ )

slope of the rising part of the EPSP is likely to be underestimated by conventional intracellular recording. Excitatory synapses are generally thought to reside mainly on the dendrites of cells (for example, on dendritic spines), whereas inhibitory synapses are closer to the somata. Therefore, the waveforms of EPSPs will show disproportionate low-pass filtering at the somatic intracellular recording site and are even faster 
relative to IPSPs then intracellular records might indicate. In addition to this biophysical reasoning, preliminary pharmacological results with nembutal and picrotoxin (presumed agonists and antagonists of cortical inhibition) appear consistent with this conclusion. ${ }^{27}$

Assuming such a hypothesis regarding the origins of field potentials, Mitzdorf and Singer ${ }^{19-21}$ were able to trace out a plausible flow of excitation through the laminae of the cat and monkey cortex with an interpretation that is consistent with many aspects of anatomy. As such, it lends further credence to their assumption regarding the centrality of excitation in generating visual field potentials. Because of its obvious importance in the interpretation of VEP origins, this hypothesis deserves greater attention and demands more experimental support. In particular, it needs to be seen whether it can be generalized to visual stimulation in addition to electrical stimulation.

\section{Concluding Discussion}

So far, I have described two approaches in the study of the VEP. Our long-range objective is to combine both approaches: to use fine stimulus control to isolate different cortical mechanisms and then to make current source density measurements to determine the anatomical location of such mechanisms. Although we have not reached this goal as yet, observations with each technique permit some tentative conclusions.

First is the finding that although there may be little in the way of a surface potential, there can be large and complex patterns of synaptic currents in the depths. The converse can also be true, namely, that a very simple set of sink/source distributions (especially if it has an "open field" configuration) can lead to large potentials on the surface. ${ }^{20}$

Given these considerations regarding the depth distribution of synaptic currents and given the "neural resonance" hypothesis mentioned earlier, one can begin to see how the steady-state VEP could be so narrowly tuned with respect to spatial and temporal frequency. Narrow spatial frequency tuning might result from a restricted set of cells which have "open field" dipole characteristics and which are also particularly sensitive to a small range of temporal and spatial frequencies.

If true, the above hypothesis presents itself as either a set of problems to be overcome and/or opportunities to be exploited. It is a problem if we wish to use the visual evoked potential as a simple one-to-one substitution for psychophysics. For example, if we wished to obtain the full contrast sensitivity function from a human observer, a single VEP amplitude versus spatial frequency function would be too biased. We could overcome this problem, however, by recording from several areas of the human scalp, and over a range of temporal frequencies, thereby obtaining an envelope of many curves which would more closely approximate the psychophysics. In fact, if we were interested in only one aspect of the contrast sensitivity curve, the upper acuity limit, for example, the technique of obtaining only one curve at one temporal frequency and at one electrode location (if properly chosen) is surprisingly good. ${ }^{28}$ As such, the selective nature of VEP recording can pose problems but they are surmountable.

What can be said for the specific opportunity afforded by VEP selectivity? Because the spatial frequency tuning of the gross VEP recorded from the scalp can be as sharp as the tuning seen for single neurons, we are presented with the tantalizing possibility that these spatial and temporal frequency peaks represent discrete neural 
entities sharing the same specific trigger features as well as a common neuronal geometry and anatomical classification.

Easy isolation of such "entities" for a given observer at a given electrode site does not come without some additional effort or without some luck, however. Field potentials, in contrast to single unit recordings, have the property of long distance propagation and summation. Thus, at any electrode site one can record voltages from anywhere eise in the brain. Therefore, it is highly likely that, unless specific care is taken, many mechanisms will be "visible" and it will be difficuit to isolate each hypothetical mechanism if the overlap is too great. In favorable cases (as shown in FIgURE 2) it appears that the isolation is rather good, but in other cases it is not. ${ }^{29}$ One possible approach to achieve a greater isolation in humans is to supplement the fine stimulus control afforded by spatial and temporal frequencies with an additional type of stimulus control, namely, by carefully restricting the area of retinal stimulation, as reported by Tyler and Apkarian in this volume. ${ }^{30}$ Another approach is to combine the use of fine stimulus control with the technique of current source density analysis in a suitable animal model. With such experiments we hope to begin the process of correlating specific surface potentials to the activity of identifiable cortical laminae and to particular cell groups.

\section{REFERENCES}

1. Desmedt, J. E., Ed. 1977. Visual Evoked Potentials in Man: New Developments. Clarendon Press, Oxford.

2. SPEKREIJSE, H. 1966. Analysis of EEG Responses in Man. Thesis. Univ. of Amsterdam, The Hague, Junk, The Netherlands.

3. Ratliff, F., J. D. Victor \& R. M. Shapley. 1978. Nonlinear analysis of visual evoked potentials in the human. J. Opt. Soc. Am. 68: 1427.

4. KULIKOWSKI, J. 1977. Separation of occipital potentials related to the detection of pattern and movement. In Visual Evoked Potentials in Man: New Developments. J. E. Desmedt, Ed. Clarendon, Oxford.

5. Stensaas, S., D. K. Eddington \& W. H. Dobelle. 1974. The topography and variability of the primary visual cortex in man. J. Neurosurgery 40: 747-755.

6. DELANGE, H. 1952. Experiments on flicker and some calculations on an electrical analogue of the foveal systems. Physica 18: 389-398.

7. CAMPBELL, F. W. \& J. G. RoBSON. 1968. Application of Fourier a nalysis to the visibility of gratings. J. Physiol. 197: 551-566.

8. Blakemore, C. \& F. W. Campbell. 1969. On the existence of neurons in the visual system selectively sensitive to the orientation and size of retinal images. J. Physiol. 203: 237260.

9. DeValois, K., D. Albrecht \& L. G. Thorell. 1978. Cortical cells: Bar and edge detectors or spatial frequency filters? In Frontiers of Visual Science. S. Cool, Ed. Springer Verlag, New York, N.Y.

10. CAMpBell, F. W. \& L. Maffei. 1970. Electrophysiological evidence for the existence of orientation and size detectors in the human visual system. J. Physiol. 207: 635-652.

11. Tyler, C. W., P. ApKARIAN \& K. NAKayama. 1978. Multiple spatial frequency tuning of electrical responses from the human visual cortex. Exp. Brain Res. 33: 535-550.

12. ApKarian, P. \& J. AdAMS. 1980. Spatio-temporal frequency tuning in alert cat VEP. Invest. Ophthal. Vis. Sci. (Suppl.): 277

13. Nakayama, K., M. MackebeN \& E. Sutter. 1980. Narrow spatial and temporal frequency tuning in the alert monkey VEP. Brain Res. 193: 263-267.

14. Schiller, P. H., B. L. Finlay \& S. F. Volman. 1976. Quantitative studies of single-cell properties in monkey striate cortex. III. Spatial frequency. J. Neurophysiol. 39: 1334 1351. 
15. VAN ESSEN, D. C. \& S. M. ZEKI. 1978. The topographic organization of rhesus monkey prestriate cortex. J. Physiol. 277: 193-226.

16. PITTs, W. 1952. Investigations on synaptic transmission. In Cybernetics Trans. 9th Conf. H. von Foerster, Ed., pp. 159-163. Josiah Macy Foundation, New York, N.Y.

17. Nicholson, C. \& J. Freeman. 1975. Theory of current source density analysis and determination of conductivity tensor for anuran cerebellum. J. Neurophysiol. 38: 356368.

18. Freeman, J. A. \& C. Nicholson. 1975. Experimental optimization of current source density techniques for anuran cerebellum. J. Neurophysiol. 38: 369-382.

19. MITZDORF, U. \& W. SinGER. 1977. Laminar segregation of afferents to lateral geniculate nucleus of the cat. An analysis of current source density. J. Neurophysiol. 40: 12271244.

20. Mitzdorf, U. \& W. Singer. 1978. Prominent excitatory pathways in the cat visual cortex (A17 and A18): A current source density analysis of electrically evoked potentials. Exp. Brain Res. 33:371-394.

21. MitZDORF, U. \& W. Singer. 1979. Excitatory synaptic ensemble properties in the visual cortex of the macaque monkey: A current source density analysis of electrically evoked potentials. J. Comp. Neurol. 187: 71-84.

22. Nicholson, C. \& R. Llinas. 1975. Field potentials in the alligator cerebellum and theory of their relationship to Purkinje cell dendritic spikes. J. Neurophysiol. 38: 356-368.

23. Vaughan, H. G. 1982. Ann. N. Y. Acad. Sci. This volume.

24. Bowling, D. \& C. MiChaEL. 1980. Projection patterns of single physiologically characterized optic tract fibers in the cat. Nature 286: 899-902.

25. LORENTE DE NO, R. 1947. A study of nerve physiology, Part 2. Rockefeller Inst. Med. Res. 132.

26. Mackeben, M., U. Mitzdorf \& K. Nakayama. (Unpublished observations).

27. Mitzdorf. U. (Personal communication).

28. Tyler, C. W., P. ApKarian, D. Levi \& K. NaKayama. 1979. Rapid assessment of visual function: An electronic sweep technique for the pattern visual evoked potential. Invest. Ophthal. 18: 703-713.

29. Apkarian, P., K. Nakayama \& C. W. Tyler. 1981. Binocularity in the human visual evoked potential: Facilitation, summation, and suppression. EEG Clin. Neurophysiol. 51: $32-48$.

30. Tyler, C. W. \& P. Apkarian. 1982. Ann. N.Y. Acad. Sci. (this volume).

31. Nakayama, K., P. Apkarian \& C. W. Tyler. 1982. Human visual evoked potentials. Isolation of cortical populations tuned to spatial frequency. In Neurophysiology and Psychology. E. Donchin, Ed. Academic Press, New York, N.Y. (in press).

32. DeValois, R. L., H. Morgan \& D. M. Snodderly. 1974. Psychophysical studies of monkey vision. III. Spatial luminance contrast sensitivity tests of macaque and human observers. Vis. Res. 14: 75-81.

33. Nakayama, K. \& M. Mackeben. Steady state visual evoked potentials in the alert primate Vis. Res. (submitted).

\section{Discussion of the Paper}

I. BODIS-WOLLNER: You have shown that VEP amplitude as a function of contrast is a complicated function. It seems to me that it may represent not one, but two or even more mechanisms, such as different ganglion cell type responses as they are reflected at the cortex. Along this line of thinking, have you attempted to relate your data to the 
mechanism Dr. Shapley described in the LGN? It occurs to me that he also described a "speeding up of the Y ganglion cell responses of the retina as a function of contrast" which leads me to the question of whether or not you considered VEP phase versus contrast curves.

K. NaKayama: We have done that, and we do see a speeding up right off. At the moment though, I do not know quantitatively how much the response speeds up. 